\title{
Deformation process modeling of the rock mass with spherical cavity created by explosion
}

\author{
Irina Frolova* \\ National Research Moscow State University of Civil Engineering, 129337, Moscow, Russia
}

\begin{abstract}
The article discusses the solution to the axisymmetric problem of determining the stresses and displacements arising from the joint action on the asymmetric load (soil resistance) array and a centrally symmetric temperature field. The case of continuous one-dimensional inhomogeneity is considered, when the deformation characteristics of a rock mass with a spherical cavity obtained by explosion are the continuous functions of one of the coordinates - the radius. The solution of the original system of equations for the displacement components is sought in the form of expansions in Fourier series in Legendre polynomials. Using the method of variablesseparation, it is possible to reduce the problem to a system of two ordinary differential equations with variable coefficients, which is solved numerically. The stress state calculation of homogeneous and inhomogeneous massifs surrounding a spherical cavity has been carried out. A comparative analysis of the results obtained has been performed.
\end{abstract}

\section{Introduction}

A rock mass containing a spherical cavity obtained by an explosion is considered. When creating underground cavities with the help of a nuclear explosion, a large amount of heat is almost instantly released in the cavity, while significant heating of the massif zone closest to the cavity occurs, followed by heat propagation into the depth of the massif and cavity surfacecooling.

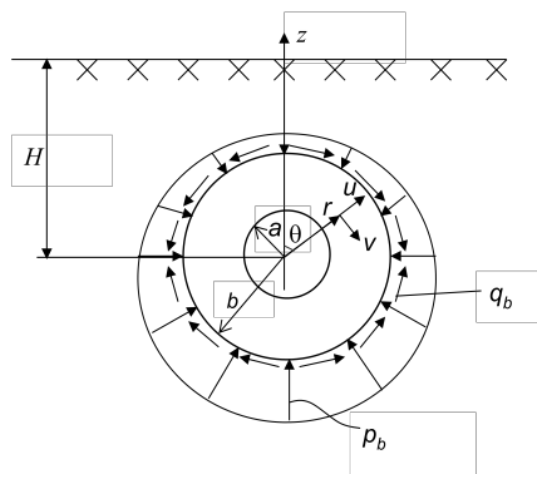

Fig. 1. Design scheme of the rock mass

*Corresponding author: ifrolova51@inbox.ru 
This process is unsteady, but, excluding the initial period from consideration and taking into account the relatively slow redistribution of the thermal field during the subsequent sufficiently long time, the problem of determining the temperature stresses can be considered as quasi-stationary.

\section{Materials and methods}

The calculation scheme of the problem is shown in Fig. 1. The calculation was carried out taking into account the real operating conditions of the massif (the presence of resistance of the medium) and the dependence of the mechanical characteristics of the soil, both on temperature and on the fracturing of the massif.

As it is known [1], with the explosive method of the underground cavities'formation, as well as with the drilling-and-blasting method of driving wells and tunnels, the surrounding rock mass undergoes such changes (the appearance of microcracks, compaction, sintering), which lead to mechanical heterogeneity of the material. In this case, a local change in the elastic modulus, which has a central symmetry, is observed. When solving the problem of thermo-elasticity at high temperature gradients, it is necessary to take into account the dependence of the elastic modulus on temperature.

So, the complete dependence $E(r)$ is presented in the form $[2,3]$ :

$$
E(r)=E_{0} e^{-\delta T}\left[1+\left(k_{1}-1\right)\left(\frac{a}{r}\right)^{m}\right]
$$

Where $k_{1}$ and $m$ are the empirical coefficients, $r$ is cavity radius.

Here $E_{0}$ is the value of the modulus of elasticity at $T={ }^{\circ} C$,

$\delta$ denotes the empirical parameter.

To assess the stress state of the massif under the influence of temperature fields and external loads, it is possible to use the equations of equilibrium in displacements [4]:

$$
\begin{aligned}
& \mu \nabla^{2} u+3(\lambda+\mu) \frac{\partial \varepsilon_{a v .}}{\partial r}-\frac{2 \mu}{r^{2}}\left(u+\frac{\partial v}{\partial \theta}+v c t g \theta\right)+3 \varepsilon_{a v} \frac{\partial \lambda}{\partial r}+2 \frac{\partial \mu}{\partial r} \frac{\partial u}{\partial r}+ \\
& +\frac{1}{r} \frac{\partial \mu}{\partial \theta}\left(\frac{1}{r} \frac{\partial u}{\partial \theta}+\frac{\partial v}{\partial r}-\frac{v}{r}\right)-3 \frac{\partial}{\partial r}\left(k \varepsilon_{\theta}\right)=0 \\
& \mu \nabla^{2} v+3 \frac{(\lambda+\mu)}{r} \frac{\partial \varepsilon_{a v .}}{\partial \theta}+\frac{\mu}{r^{2}}\left(2 \frac{\partial u}{\partial \theta}-\frac{1}{\sin ^{2} \theta} v\right)+\frac{3}{r} \frac{\partial \lambda}{\partial \theta} \varepsilon_{a v .}+\frac{\partial \mu}{\partial r}\left(\frac{1}{r} \frac{\partial u}{\partial \theta}+\frac{\partial v}{\partial r}-\frac{v}{r}\right)+ \\
& +\frac{2}{r^{2}} \frac{\partial \mu}{\partial \theta}\left(u+\frac{\partial v}{\partial \theta}\right)-\frac{3}{r} \frac{\partial}{\partial \theta}\left(k \varepsilon_{\sigma}\right)=0 .
\end{aligned}
$$

Here:

$$
\nabla^{2}=\frac{1}{r^{2}} \frac{\partial}{\partial r}\left(r^{2} \frac{\partial}{\partial r}\right)+\frac{1}{r^{2} \sin \theta} \frac{\partial}{\partial \theta}\left(\sin \theta \frac{\partial}{\partial \theta}\right)
$$


$\lambda(r), \mu(r)$ are Lamé constants; $k(r)$ denotes the bulk modulus; $\varepsilon_{6}(r)=\alpha T(r)$ means forced thermal deformations; $\alpha=$ const is a linear thermal expansion coefficient;

$v$ defines Poisson's ratio.

The boundary conditions are:

on the inner surface $(r=a) \begin{array}{r}\sigma_{r}=0, \\ \tau_{r \theta}=0 ;\end{array}$

on the outer surface $(r=b)$ $\sigma_{r}=-p_{b}(\theta)$,

$$
\tau_{r \theta}=q_{b}(\theta)
$$

Where $p_{b}$ and $q_{b}$ - surface loads, given functions from $\theta$ [5]:

$$
\begin{aligned}
& p_{b}(\theta)=\gamma(H-b \cos \theta)\left(\frac{v}{1-v}+\frac{1-2 v}{1-v} \cos ^{2} \theta\right) \\
& q_{b}(\theta)=\frac{1}{2} \gamma(H-b \cos \theta) \frac{1-2 v}{1-v} \sin 2 \theta
\end{aligned}
$$

The solution of the system (Eq. 2) with boundary conditions (Eq. 3) is proposed to be sought in the form of displacementsexpansions in Fourier series:

$$
\begin{aligned}
& u(r, \theta)=\sum_{n=0}^{\infty} u_{n}(r) P_{n}(\cos \theta) \\
& v(r, \theta)=\sum_{n=0}^{\infty} v_{n}(r) \frac{d P_{n}(\cos \theta)}{d \theta}
\end{aligned}
$$

Where $P_{n}(\cos \theta)$ is the Legendre polynomial $\ln$-th degree.

Substitution of the expressions (Eq. 4) into the system (Eq. 2) gives these equations for $u_{0}$ to the equation:

$u_{0}^{\prime \prime}(\lambda+2 \mu)+u_{0}^{\prime}\left[\frac{2(\lambda+2 \mu)}{r}+\lambda^{\prime}+2 \mu^{\prime}\right]-u_{0}\left[\frac{2(\lambda+2 \mu)}{r^{2}}-\frac{2}{r} \lambda^{\prime}\right]-3\left(k \varepsilon_{b}\right)^{\prime}=0$

and for $u_{n}$ and $v_{n}(\mathrm{n} \geq 1)$ to the system of two second-order ordinary differential equations with variable coefficients (the prime denotes differentiation along the radius):

$$
\begin{aligned}
& u_{n}^{\prime \prime}(\lambda+2 \mu)+u_{n}^{\prime}\left[\frac{2(\lambda+2 \mu)}{r}+\lambda^{\prime}+2 \mu^{\prime}\right]-u_{n}\left[\frac{\mu}{r^{2}} n(n+1)+\frac{2(\lambda+2 \mu)}{r^{2}}-\frac{2}{r} \lambda^{\prime}\right]+ \\
& +\left[-v_{n}^{\prime}\left(\frac{\lambda+\mu}{r}\right)+v_{n}\left(\frac{\lambda+3 \mu}{r^{2}}-\frac{1}{r} \lambda^{\prime}\right)\right] n(n+1)=0
\end{aligned}
$$




$$
\begin{aligned}
& v_{n}^{\prime \prime} \mu+v_{n}^{\prime}\left(\frac{2 \mu}{r}+\mu^{\prime}\right)-v_{n}\left[n(n+1) \frac{\lambda+2 \mu}{r}+\frac{1}{r} \mu^{\prime}\right]+u_{n}^{\prime} \frac{\lambda+\mu}{r}+ \\
& +u_{n}\left[\frac{2(\lambda+2 \mu)}{r^{2}}+\frac{1}{r} \mu^{\prime}\right]=0 .
\end{aligned}
$$

The calculations show that in the $\operatorname{expansions}_{b}(\theta)$ and $q_{B}(\theta)$ it is enough to limit ourselves to the members up ton $=3$ :

$$
\begin{aligned}
& p_{b}(\theta)=\sum_{n=0}^{\infty} c_{n} p_{n}(\cos \theta) \\
& q_{b}(\theta)=\sum_{n=0}^{\infty} d_{n} \frac{d p_{n}(\cos \theta)}{d \theta} .
\end{aligned}
$$

The coefficients of these expansions $c_{n}$ and $d_{n}$ are determined by the well-known formulas [4].

Thus, in (Eq. 6) the systems for $n=1 \div 3$.

Further solution of the system (Eq. 6) with arbitrary dependences $\lambda(r), \mu(r)$ and $k(r)$ should be done numerically. Various ways of numerical implementation of the considered equations are possible. In this paper, we use the matrix orthogonal sweep method[6].

Having defined $u$ and $v$, the stresses $\sigma_{r}, \sigma_{\theta}, \sigma_{\varphi}$ and $\tau_{r} \theta$ can be calculated by the formulas:

$$
\begin{aligned}
\sigma_{r} & =\sum_{n=0}^{3}\left[\frac{2 \lambda}{r} u_{n}+(\lambda+2 \mu) u_{n}^{\prime}-\frac{\lambda n(n+1)}{r} v_{n}-3 k g_{n}\right] P_{n}(\cos \theta) ; \\
\sigma_{\theta} & =\sum_{n=0}^{3}\left\{\left[\frac{2(\lambda+2 \mu)}{r} u_{n}+\lambda u_{n}^{\prime}-\frac{\lambda n(n+1)}{r} v_{n}-3 k g_{n}\right] P_{n}(\cos \theta)+\frac{2 \mu}{r} v_{n} \frac{d^{2} P_{n}(\cos \theta)}{d \theta^{2}}\right\} ; \\
\sigma_{\varphi} & =\sum_{n=0}^{3}\left\{\left[\frac{2(\lambda+2 \mu)}{r} u_{n}+\lambda u_{n}^{\prime}-\frac{\lambda n(n+1)}{r} v_{n}-3 k g_{n}\right] P_{n}(\cos \theta)+\frac{2 \mu}{r} v_{n} \operatorname{ctg} \theta \frac{d P_{n}(\cos \theta)}{d \theta}\right\} ; \\
\tau_{r \theta} & =\sum_{n=1}^{3} \mu\left[\frac{1}{r} u_{n}-\frac{1}{r} v_{n}+v_{n}^{\prime}\right] \frac{d P_{n}(\cos \theta)}{d \theta} .
\end{aligned}
$$

Where $g_{0}=\alpha T(r)$ at $n=0, g_{n}=0$ at $n \neq 0$.

\section{Calculation results}

With the help of the developed program, the calculations were carried out with the following initial data: $a=25 \mathrm{~m} ; 6=250 \mathrm{~m}$;

$H=1200 \mathrm{~m} ; \gamma=2.1510^{3} \mathrm{~kg} / \mathrm{m}^{3} ; E_{0}=210^{4} \mathrm{MPa} ; v=0.23$.

Temperature propagation in an isotropic medium under the action of instant heat sources distributed over a sphere of radius $a$ according to[1]is described by the formula:

$$
T(r, t)=\frac{Q_{0}}{8 \pi \sqrt{\pi} c \gamma a r \sqrt{a_{1} t}} e^{-\frac{(r-a)^{2}}{4 a_{1} t}}+T_{1}
$$


Where $T_{1}$ is an initial array temperature; $T$ is the temperature at a point spaced from the center of the cavity at a distance $r \geq a$, at the moment $t ; Q_{0}$ is the amount of heat generated;

$c$ is the specific heat of the medium; $\gamma$ denotes density; $a_{1}=\lambda / c$ isthermal diffusivity;

$\lambda$ is the material thermal conductivity coefficient; $a$ defines cavity radius.

The calculations used the averaged thermophysical characteristics of the soil are: $c=0.96 \mathrm{~kJ} /($ kgdegree $) ; a_{1}=1.6710^{-6} \mathrm{~m}^{2} / \mathrm{s}$.

Fig. 2 shows the temperature curves for some points in time $t$ [7]:

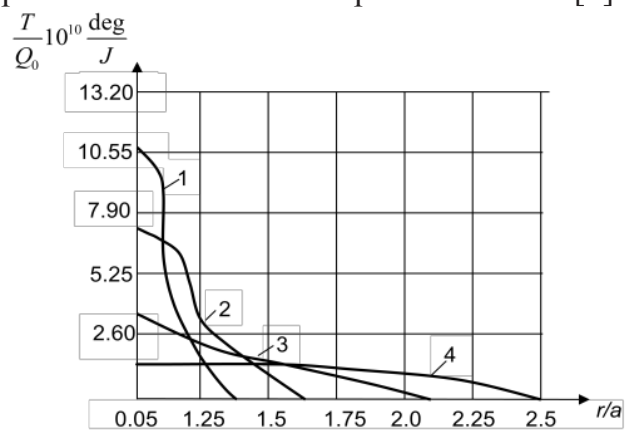

Fig. 2. Temperature curves for the points in time: $1-3.610^{6} \mathrm{~s} . ; 2-7.210^{6} \mathrm{~s} . ; 3-4.02510^{7} \mathrm{~s} . ; 4-1.61$ $10^{8} \mathrm{~s}$.

It is of considerable interest to study the temperature inhomogeneity effect on the rock mass stress state temperature components values $[8,9,10]$.

Fig. 3 shows the diagrams of temperature stresses $\sigma_{\theta}$ for a moment in time $t=1.6110^{8} \mathrm{~s}$. In this case, the parameter characterizing the fracturing of the rock $k_{1}=1$. The graphs show a significant change in stresses near the contour in an inhomogeneous material in comparison with a homogeneous material, which is associated with a significant drop in the elastic modulus due to temperature inhomogeneity.

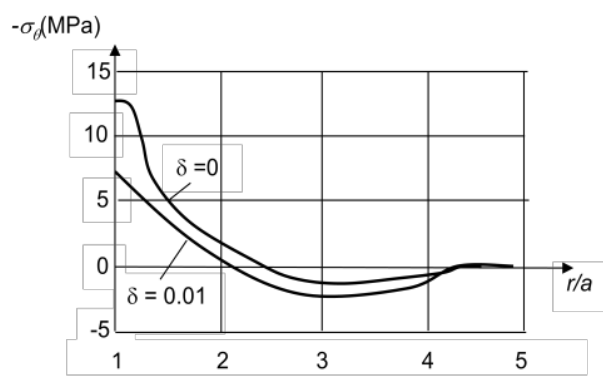

Fig. 3. Diagrams of temperature stresses fort $=1.6110^{8} \mathrm{~s} .\left(\theta=90^{0}, \mathrm{k}_{1}=1\right.$, $)$

Fig. 4 shows diagrams of total stresses $\sigma_{\theta}$ (at $\theta=90^{\circ}$ ), caused by the simultaneous action of temperature and force loads on the rock mass under consideration, taking into account both types of material inhomogeneity. 


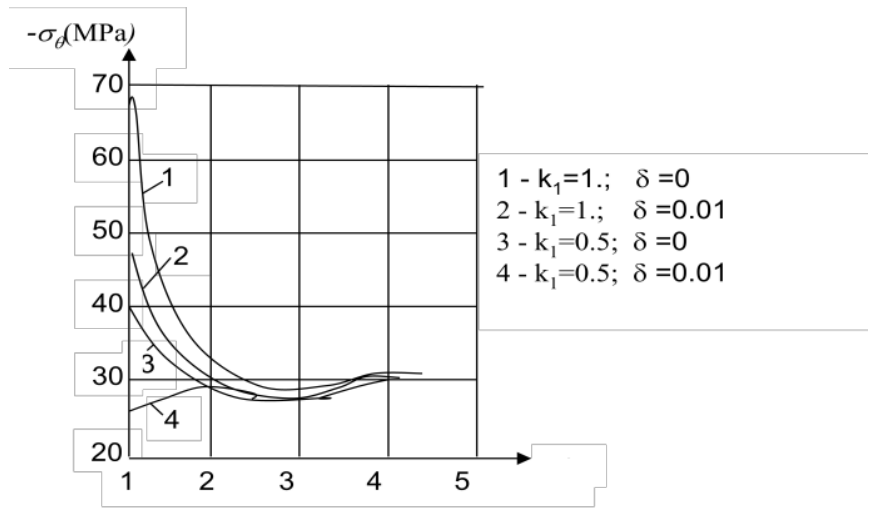

Fig. 4. Diagrams of total stresses $\left(\theta=90^{0}, t=1.6110^{8} \mathrm{~s}\right.$.

It can be seen from the graphs that taking into account two types of inhomogeneity leads to an even more significant reduction in stresses, especially in the immediate vicinity of the cavity contour. Here, this voltage difference is approximately $50 \%$. Further, when moving away from the contour of the cavity at a distance equal to $2 a$ the results of calculations in the inhomogeneous and homogeneous cases practically coincide. A similar trend is observed when solving similar problems by numerical methods $[11,12]$.

Change in voltages $\sigma_{\theta}$ along the contour of the cavity is shown in Fig. 5. It can be seen from these graphs that the stresses differ significantly in the entire range of values $\theta$ for homogeneous and heterogeneous arrays. The exceptions are the zones close to the cavity poles $\left(\theta=0^{\circ}\right.$ and $\theta=180^{\circ}$ ). The largest difference in voltage values is observed at $\theta=90^{\circ}$. But when approaching the poles, this difference becomes less and less noticeable.

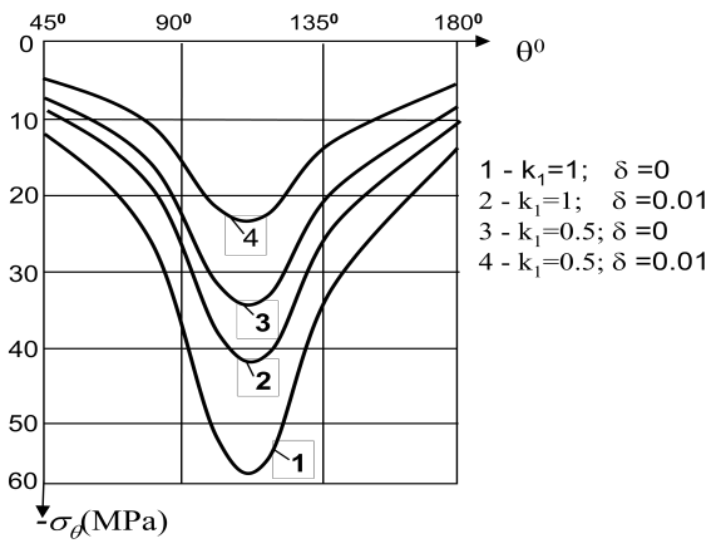

Fig. 5. Diagrams of stresses along the contour of the cavity for $t=1.6110^{8} \mathrm{~S}$

\section{Conclusion}

Analyzing the obtained results of the calculations, we can conclude that taking into account the heterogeneity of the material is necessary when calculating rock masses under power loads and under temperature effects that inevitably arise when the cavities are created in them by means of explosion. 


\section{References}

1. A.A.Bakirov, Application of underground nuclear explosions in the petroleum industry (Nedra, Moscow, 1981).

2. A.O. Vatulyan, Bulletin of the Samara state. University 4 (54), 93-103 (2007).

3. I.I. Frolova, M.K. Agakhanov, E3S Web of Conferences 33, 194-199 (2018).

4. V.I. Andreev, Some problems and methods in mechanics of inhomogeneous bodies (ASV, Moscow, 2002).

5. A.T. Vasilenko, Ya.M. Grigorenko, Applied mechanics 28, 22-26 (1982).

6. A.A. Samarskiy, E.S. Nikolaev, Methods for solving grid equations (Nauka, Moscow, 1978).

7. I.I. Frolova, IOP Conf. Ser.: Mater. Sci. Eng. 661, 012094 (2019)

8. V.I. Andreev, S. V. Bulushev, Vestnik of Moscow State University of Construction 12, 40-46 (2012).

9. V.I. Andreev, A.S. Avershyev, Procedia Engineering 111, 30-35 (2015).

10. V.I. Andreev, A.S. Avershyev, Construction mechanics of engineering structures and structures (PFUR) 1, 30-36 (2014).

11. I.I. Frolova, V.I. Andreev, Materials Science Forum 931, 78-83 (2018).

12. I.I. Frolova, IOP Conf. Ser. Mater. Sci. Eng. 913, 022037 (2020). 